\title{
NEW RESULTS ON ELECTRON BROADENING OF SOME \\ UV LINES OF NII, CII/IV AND SiII/III/IV
}

\author{
S. SAHAL-BRECHOT and E. SEGRE \\ Observatoire de Paris Meudon, 92 Meudon, France
}

\begin{abstract}
The electron damping constants of some UV lines of astrophysical interest are calculated within the impact and semiclassical approximations and are compared with experimental results where these are available (NII, UV(1) and CII, UV (1)).
\end{abstract}

The good agreement between experimental results and the semi-classical calculations of Sahal-Brechot (1969a, b) and Chapelle and Sahal-Brechot (1970) has led us to perform other calculations on UV lines of astrophysical interest. First we give a short account of our method. The widths are calculated within the impact approximation (Baranger, 1958). Under this condition the profile is Lorentzian and the width is given by the quantum formula (77c) of Baranger (1958). The validity criterion is

$$
\gamma \tau \ll 1
$$

where $y$ is the width of the line (damping constant) and $\tau$ a typical collision time. We have shown (Sahal-Brechot, 1969a, b; Chapelle and Sahal-Brechot, 1970) that the impact approximation is valid for both electron and proton collisions in stellar atmospheres. For this reason we have also calculated the damping constants for proton and $\mathrm{He}^{+}$collisions.

The scattering amplitudes and cross-sections which interfere in the calculation of the width are evaluated within the semi-classical approximation (Alder et al., 1956); first, the perturber is assumed to be a classical particle moving along a classical path unperturbed by the collision; for radiating ions this path is a hyperbola. This is the main improvement compared with the Baranger-Griem calculations (Griem et al., 1962; Griem, 1964); the use of a hyperbolic path instead of a straight one increases the resulting widths by a factor between 3 and 5 . Secondly, the second order perturbation theory for the interaction electrostatic potential is used; dipole inelastic interactions, dipole (polarization) and quadrupole $(20 \%$ of the total width) elastic interactions are taken into account; the quadrupole inelastic interactions are negligible; the inelastic interactions are important only for electron collisions. Symmetrized cross-sections (Alder et al., 1956; Seaton, 1962) are used; the impact parameter cut-off is chosen in a way which copes with the conservation of the flux of particles, i.e. the $S$-matrix is unitary (Seaton, 1962).

The semi-classical approximation is obviously valid when most of the collisions are weak, i.e. the close (or strong) collisions are not important. The validity criterion is the following;

$$
\Delta E / k T \ll 1
$$


where $\Delta E$ is a typical energy gap between the interacting levels of the radiating atom and $k T$ is the temperature. Nevertheless the comparisons between semi-classical calculations, experiments and the more recent elaborate quantum calculations (Bely and Griem, (1970) for MgII 3s-3p, Barnes and Peach (1970) for CaII $4 s-4 p$ ) show that the semi-classical calculations are accurate to within $30 \%$, despite the importance of the strong collisions $\left(75 \%\right.$ in the case of $\mathrm{Mg}^{+} 3 s-3 p$ where $\Delta E / k T>4$ ). This agreement probably comes from the care taken with the physical nature of the model (use of symmetrized cross-sections and a unitary $S$-matrix).

From the results presented in this paper it can be seen that our calculations for the UV resonance lines $C_{I I}$ (1) and $\mathrm{N}_{\text {II }}$ (1) (Figures 1 and 2) fit the recent experiments of Fortna (1969) and yet the typical energy gap for these two lines is of the order of $10 \mathrm{eV}$ ! The close collisions are predominant and the typical impact parameter $\varrho_{\text {eff }}$ is very small (see Table I). Table I shows also that the impact approximation is valid for both electrons and protons: $\gamma$ is the calculated (or experimental) value of the damping constant for a density, $N_{e}=10^{15} \mathrm{~cm}^{-3}$, and $T=2 \times 10^{4} \mathrm{~K}$; the typical collision

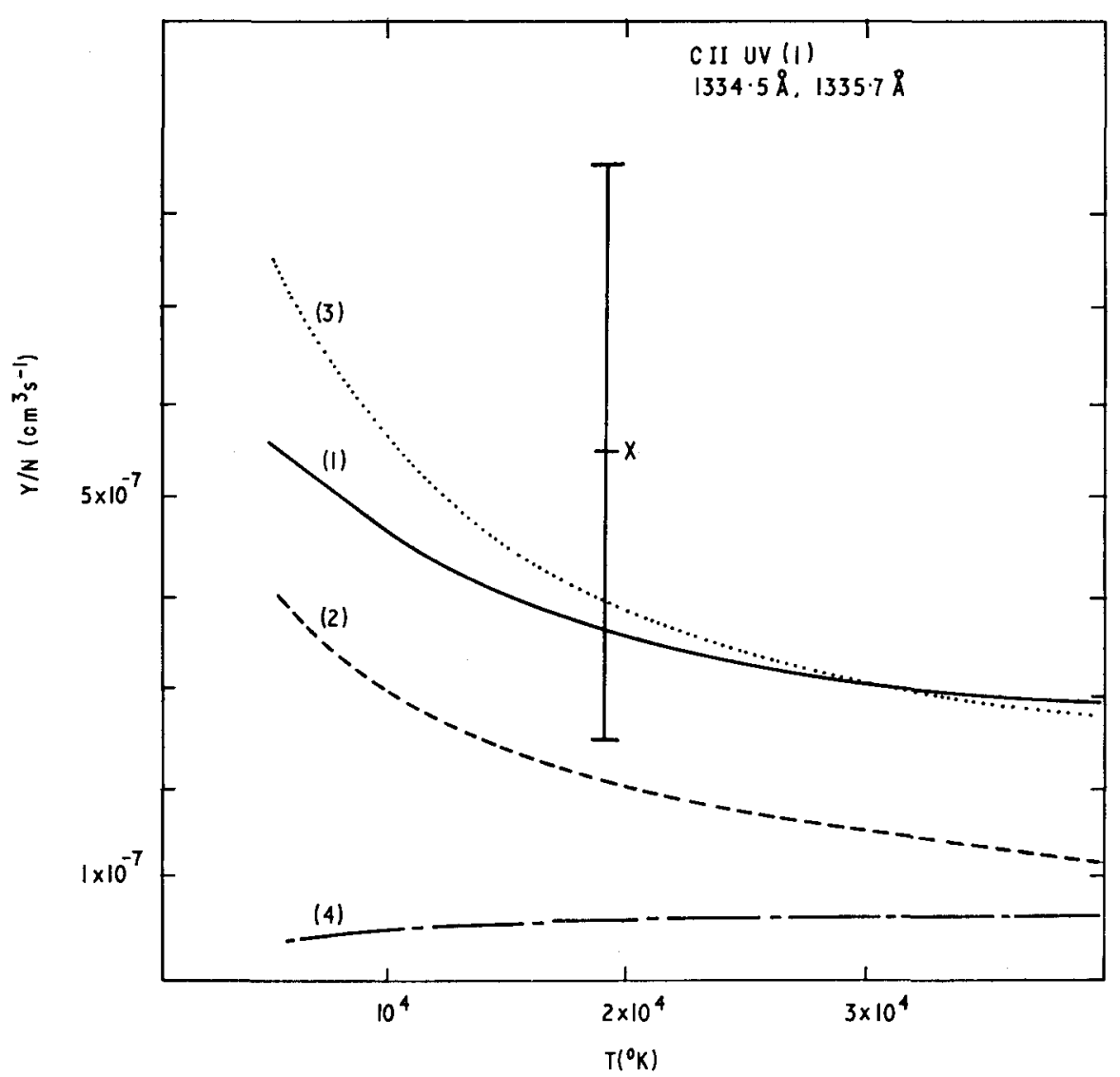

Fig. 1. 


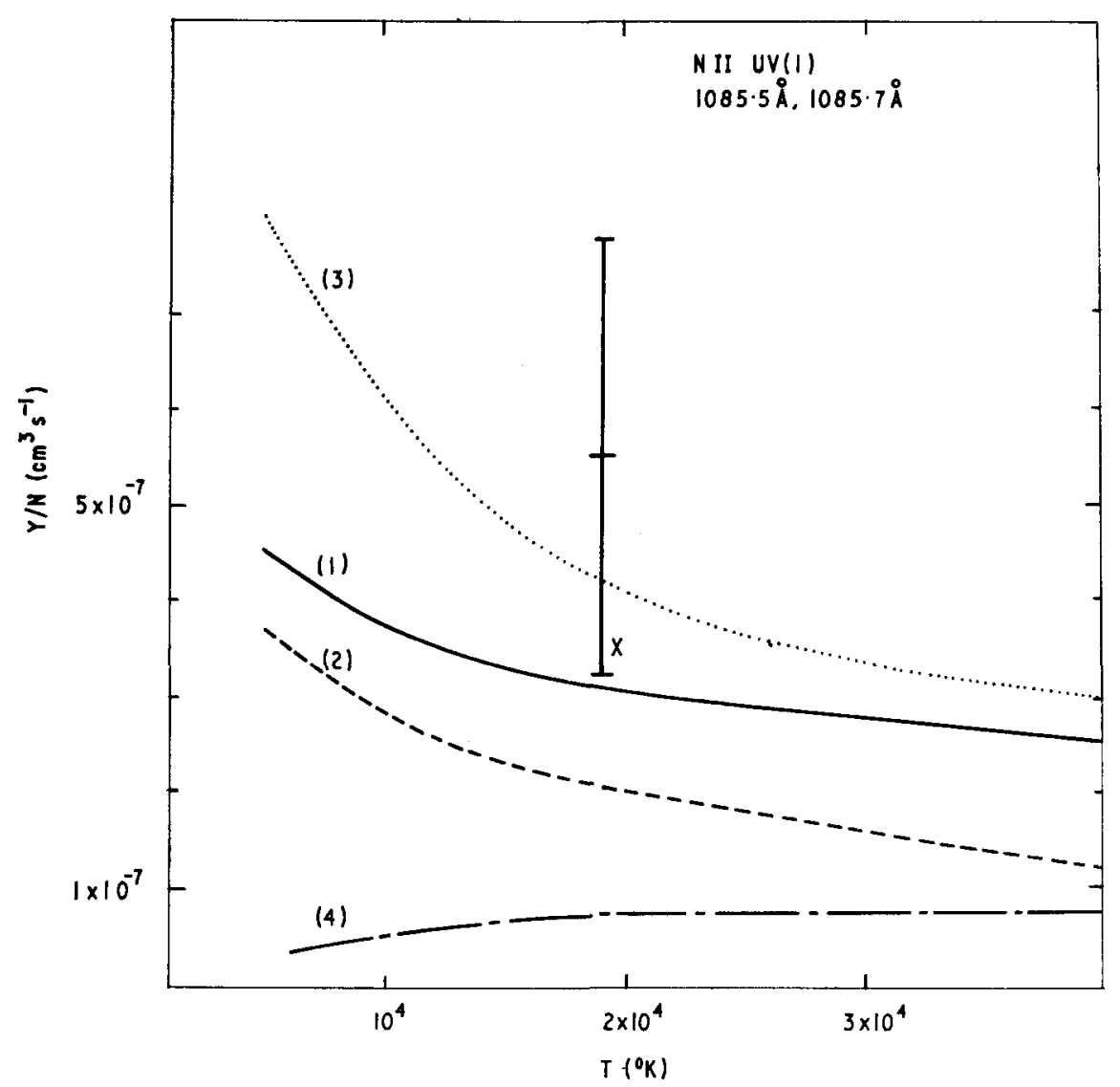

Fig. 2.

Fig. 1-2. Electron Damping constant of CII, UV (1) and NII, UV (1) (for a density of $1 \mathrm{~cm}^{-3}$ ) versus the electronic temperature. Curve (1) this work. (2) this work, elastic collisions only. (3) Formula (102) of Brechot and Van Regemorter (1964) (No (1) of this paper). (4) Lindholm (1942). $\times$ semiempirical (Griem, 1968). Classical damping constant: experiment of Fortna (1969)

$\left\{1.2 \times 10^{9}\right.$ for CII (1)

$\left\{1.9 \times 10^{9}\right.$ for $\mathrm{N}$ II (1)

TABLE I

\begin{tabular}{|c|c|c|c|c|c|c|c|c|}
\hline & \multicolumn{2}{|c|}{$\varrho_{\mathrm{ePf}} / a_{0}$} & \multirow[t]{2}{*}{$\Delta E \mathrm{eV}$} & \multirow{2}{*}{$\begin{array}{l}\text { close coll. } \\
\text { contrib. }\end{array}$} & \multicolumn{2}{|c|}{$l$} & \multicolumn{2}{|c|}{$\gamma \tau$} \\
\hline & electrons & protons & & & electrons & protons & electrons & protons \\
\hline CII UV (1) & 6 & 10 & 9 & $80 \%$ & 1 to 2 & 125 & $3 \times 10^{-7}$ & $2 \times 10^{-5}$ \\
\hline NII UV (1) & 4 & 6 & 11 & $80 \%$ & 1 & 75 & $2 \times 10^{-7}$ & $1 \times 10^{-5}$ \\
\hline
\end{tabular}

Qeff typical impact parameter for $T=2 \times 10^{4} \mathrm{~K}$

$a_{0}$ Bohr radius

$\Delta E$ typical energy difference between the interacting levels

$l$ typical angular momentum of the perturber

$\gamma \quad$ damping constant for $N_{\mathrm{e}}=10^{15} \mathrm{~cm}^{-3}$ and $T=2 \times 10^{4} \mathrm{~K}$

$\tau$ typical collision time for $T=2 \times 10^{4} \mathrm{~K}$ 


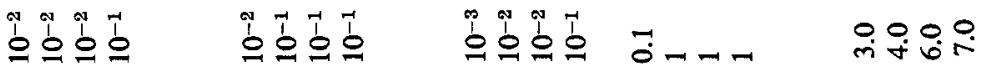

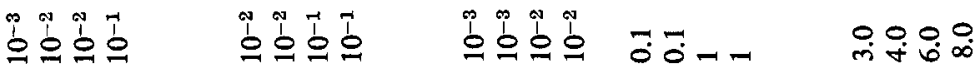

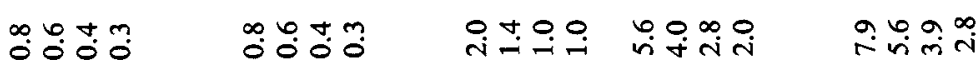

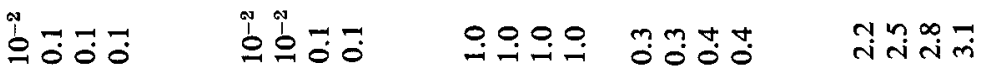<smiles>C=CC</smiles>

$\stackrel{n}{\sim}$

已

u

$\stackrel{0}{\circ} \stackrel{0}{-}$

$\stackrel{9}{m}$

$\bar{z}$<smiles>C1CCCC1</smiles>

प웡웡

흉융웅융

등으늉웅

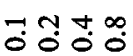

竞

๖ณุ์

ำถำำ

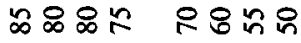

$888 \infty$

ตัติำ

ํํㅇ융

mơ

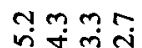

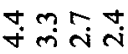

$\tilde{c}-n+$

$\tilde{0}-N+$

$\frac{0}{n-4} \pi \tilde{0}-4$

$\tilde{0}-n d$

क

ลิ

สู

î.

童
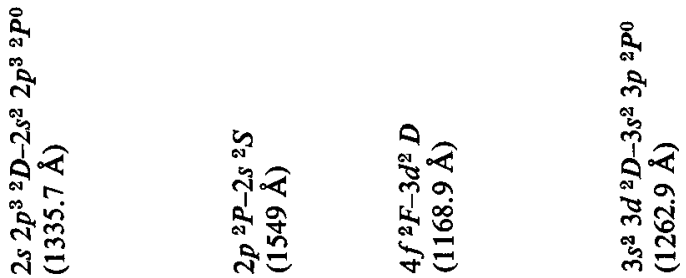
.

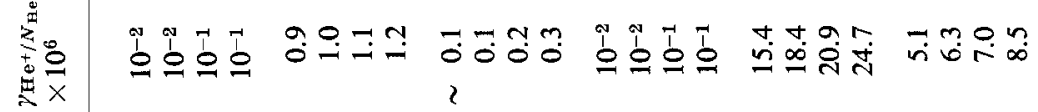

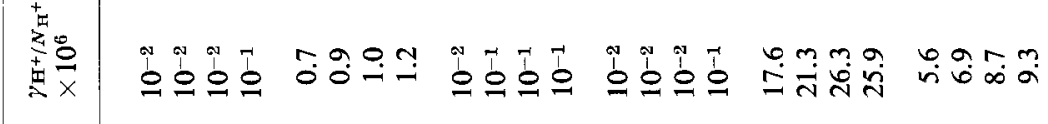

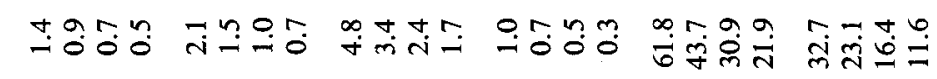

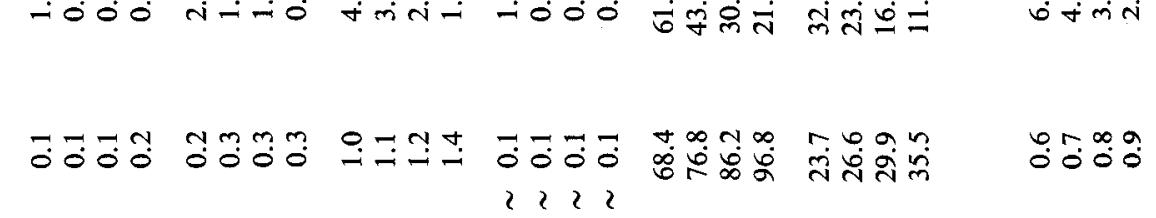

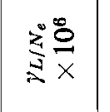

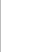

$\stackrel{\circ}{x}$ (1)

$\underset{2}{2} \frac{2}{5}$

$\underline{\sigma}$

$\stackrel{2}{\sigma}$

$\stackrel{5}{0} \quad \stackrel{n}{0}$

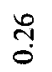

宅产追:

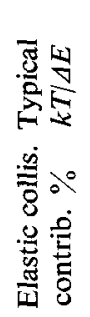

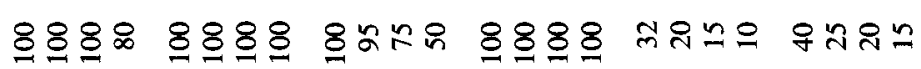

888

$\stackrel{2}{2} \stackrel{\stackrel{\circ}{x}}{x}$

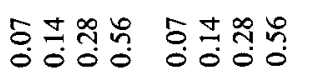

ํㅜㅇ 苍茴

$\stackrel{8}{0}$

능요요융요

春

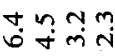

$\stackrel{4}{\stackrel{4}{4}}$

융요요

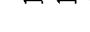

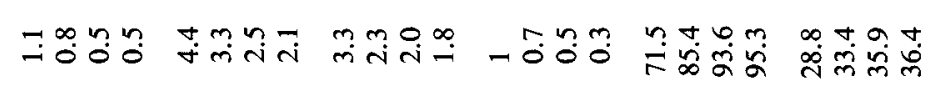

$\mathfrak{n}=\stackrel{m}{-\infty}$

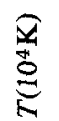

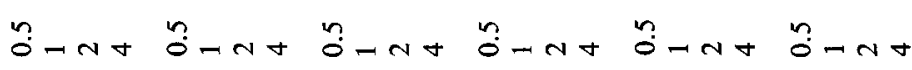

$\tilde{m}-4 t$

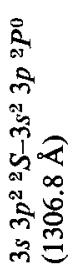

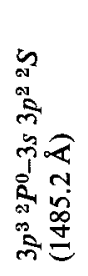

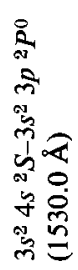

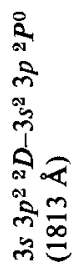

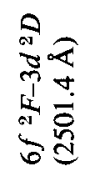

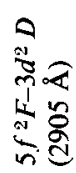

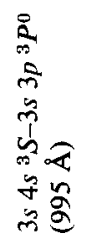




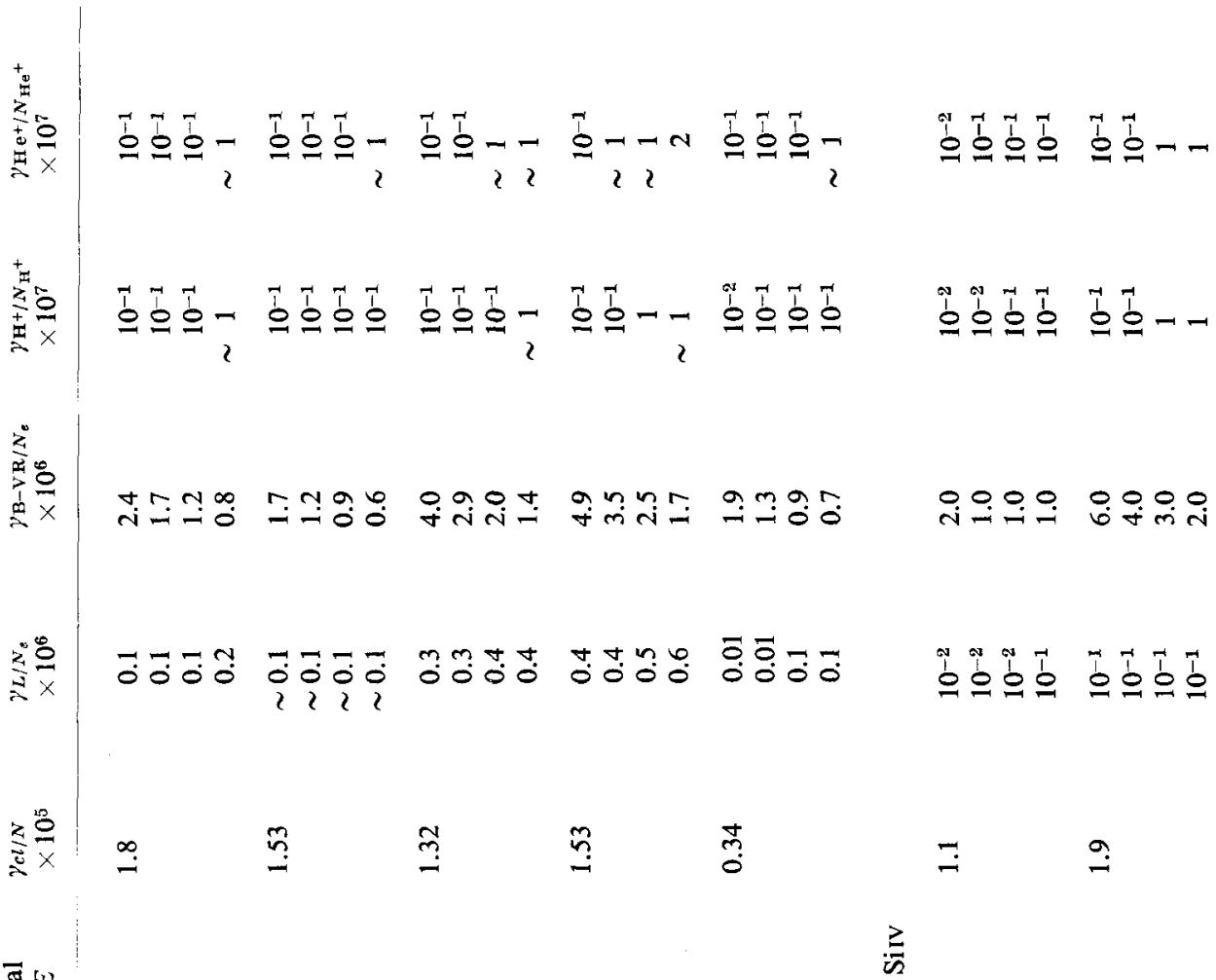

垩出|

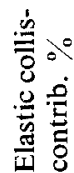

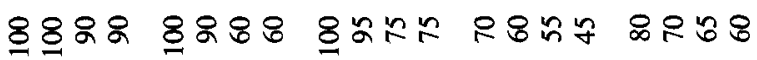

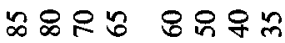

$\sum^{\circ} \stackrel{\circ}{=}$

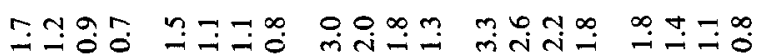

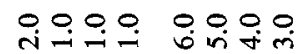

x

\section{पथ}

ก

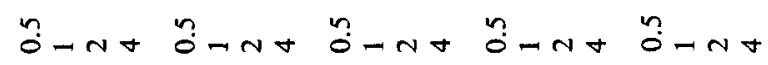

$\tilde{0}-n+\tilde{0}-n+$

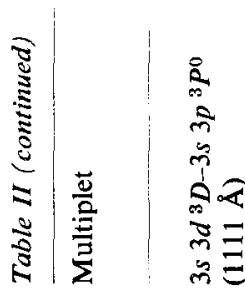

$\stackrel{\infty}{\circledR}$

के

$\stackrel{m}{\dot{\theta}}$

인

$\stackrel{m}{m \Xi}$

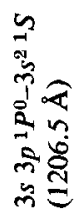

㐫

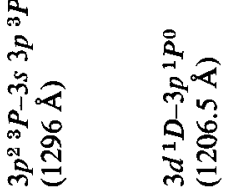

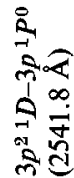

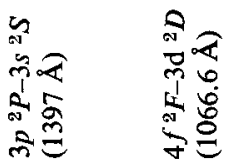




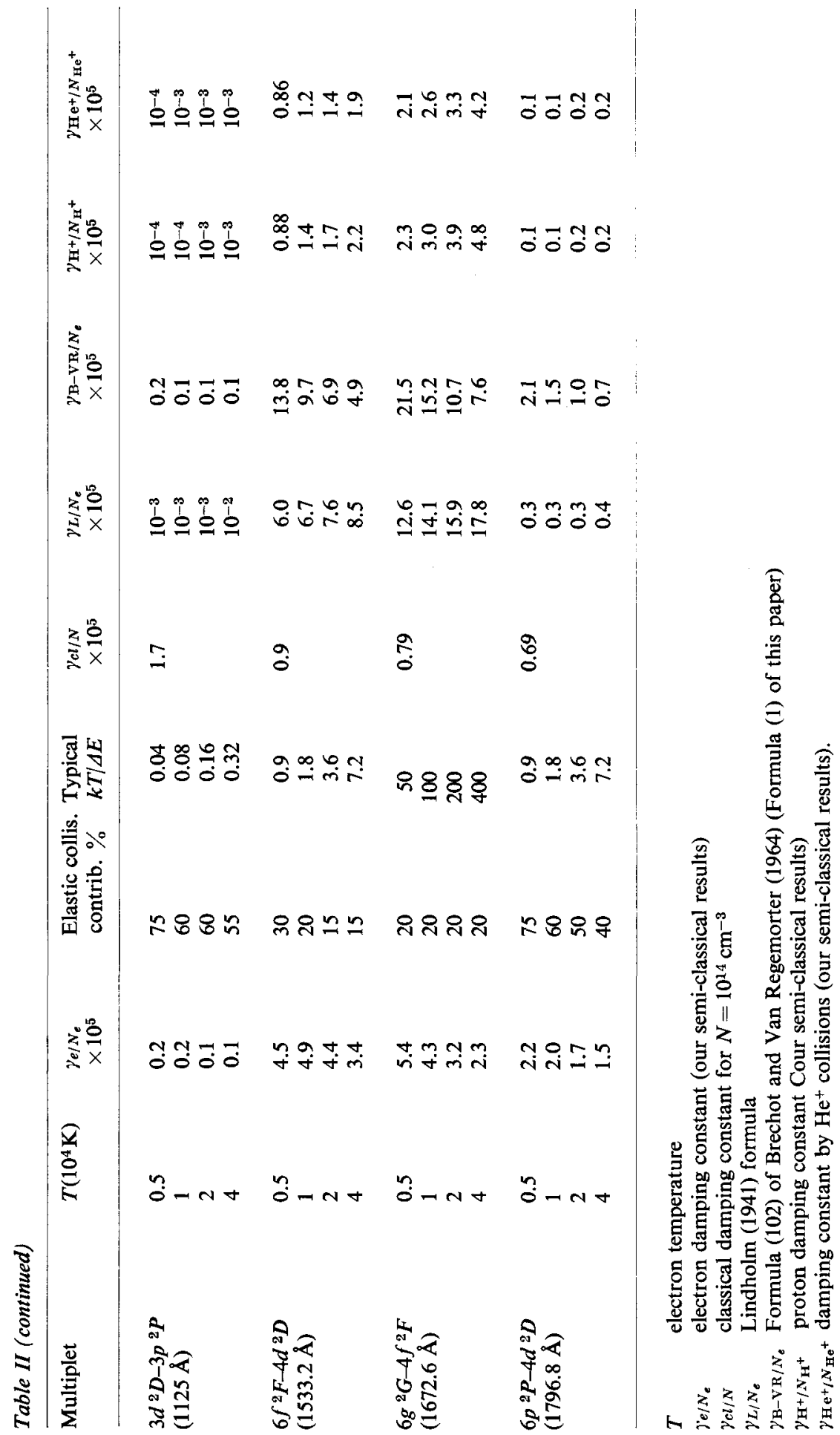


time, $\tau$ is of the order of $\varrho_{\text {eff }} / v$; the velocity of the perturber $v$ is of the order of $\sqrt{ }(8 k T) /(\pi m)$ where $m$ is the reduced mass. The typical impact parameter $\varrho_{\text {eff }}$ is evaluated a posteriori (Chapelle and Sahal-Brechot, 1970) in the following manner: first, the cross-section $\sigma$ is evaluated and then the predominant impact parameter $\varrho_{\text {eff }}$ is deduced (one can write $\sigma=\pi \varrho_{\text {eff }}^{2}$ for instance).

From Figures 1 and 2 it can be seen that the semi-empirical formula of Griem (1968) and the simple formula of Brechot and Van Regemorter (1964), analogous to the Lindholm (1943) adiabatic formula but with a hyperbolic classical path,

$$
\gamma=N_{e} 3.86 \times 10^{-5} Z^{4 / 5}\left|\alpha_{i}-\alpha_{f}\right|^{2 / 5} T^{-1 / 2}
$$

also give reliable results for these two lines. $N_{e}$ is the electron density in $\mathrm{cm}^{-3}, Z$ is the charge of the radiating ion, $\alpha_{i}$ and $\alpha_{f}$ are the polarisabilities, in atomic units, of the radiating ion in the stage $i$ or $f$, the initial and final levels of the transition involved. Nevertheless, these two formulae must be used with caution if a result with accuracy better than a factor of two is required. The Lindholm (1941) formula gives a rather poor result and likewise the classical value of the damping constant gives results which are greatly overestimated at stellar densities $\left(N \approx 10^{14} \mathrm{~cm}^{-3}\right)$.

In Table II the damping constants of several SiII, III, IV, CII, IV and $\mathrm{N}_{\text {II }}$ (1) lines are collected. The typical energy difference $\Delta E$ gives the order of the uncertainty of the results. If $k T / \Delta E>10$, the uncertainty is less than $20 \%$, if $k T / \Delta E<\frac{1}{5}$, it is of the order of $30 \%$ or $40 \%$. The classical value $\gamma_{c l} / N$ (evaluated for $N_{e}=10^{14} \mathrm{~cm}^{-3}$ ), the Lindholm value (1941) and the Brechot and Van Regemorter (1964) value (formula 1 of this paper) are given for comparison.

When $k T / \Delta E \ll 1$ the simple $\mathrm{B}-\mathrm{VR}$ formula is rather useful; indeed, the elastic contribution is predominant and the adiabatic assumption is valid. Under this condition the very simple formula can give quite reliable results. Nevertheless, the semiclassical calculations are also very simple to perform, the Fortran IV program of the Observatoire de Meudon gives the result after a few seconds (IBM 360-65 computer), the input data are only the oscillator strengths and the energy differences of the interacting levels of the transitions involved.

\section{References}

Alder, K., Bohr, A., Huus, T., Mottelson, B., and Winther, A.: 1956, Rev. Mod. Phys. $28,432$.

Baranger, M.: 1958, Phys. Rev. 112, 855.

Barnes, K. and Peach, G.: 1968, J. Phys. B. (2), 3, 350.

Bely, O. and Griem, H. R.: 1970, Phys. Rev. 1A, 97.

Brechot, S. and Van Regemorter, H.: 1964, Ann. Astrophys. 27, 432.

Chapelle, J. and Sahal-Brechot, S.: 1970, Astron. Astrophys. 6, 415.

Fortna, J. D. E. : 1969, NRL Report 6950.

Griem, H. R.: 1964, Plasma Spectroscopy McGraw Hill Book Co.

Griem, H. R.: 1968, Phys. Rev. 165, 258.

Griem, H. R., Baranger, M., Kolb, A. C., and Oertel, G.: 1962, Phys. Rev. $125,177$.

Lindholm, E.: 1941, Arkiv Mat. Astron. Fysik, 28B, No. 3.

Sahal-Brechot, S.: 1969a, Astron. Astrophys. 1, 91.

Sahal-Brechot, S.: 1969b, Astron. Astrophys. 2, 322.

Seaton, M. J.: 1962, Proc. Phys. Soc. 79, 1105. 


\section{DISCUSSION}

G. K. Oertel: Elastic collisions contribute strongly to the electron broadening of ionic lines. The impact theory with hyperbolic classical path is therefore applicable to only a small portion of the total damping constant in most cases - The good agreement with other calculations and with experiment is therefore very surprising. 